\title{
Permanencia de Restauraciones Oclusales Realizadas con Téc- nica Restaurativa Atraumática (TRA) en Dientes Permanentes
}

\author{
Survival of Atraumatic Restorative Treatment (ART) Restorations in Permanent Teeth
}

Katherine Rudloff T.; Rodrigo Haristoy O."* \& Manuel Velásquez C."**

RUDLOFF, T. K.; HARISTOY, O. R. \& VELÁSQUEZ, C. M. Permanencia de restauraciones oclusales realizadas con técnica restaurativa atraumática (TRA) en dientes permanentes. Int. J. Odontostomat., 8(1):53-58, 2014.

RESUMEN: El tratamiento restaurador atraumático (TRA) ha sido incorporado a la práctica clínica como un método definitivo o provisional para controlar los altos niveles de caries. Es de especial utilidad cuando se necesita prestar atención a comunidades aisladas con difícil acceso al sistema de salud público. El objetivo fue determinar la permanencia y estado de restauraciones realizadas con técnica restaurativa atraumática en dientes permanentes de niños entre 6 - 16 años en la comuna de Curarrehue, Región de la Araucanía, Chile. La población blanco la conformaron 249 niños entre 3 y 16 años residentes en la ciudad de Curarrehue. Luego de 7 meses de realizadas las obturaciones se realizó un examen clínico a los participantes, donde se evaluaron las restauraciones de acuerdo a los criterios establecidos por Frencken para la evaluación del TRA. Se examinaron 172 dientes restaurados con TRA; 142 estaban presentes exitosamente, correspondiente a un $82 \%$ de permanencia. En relación al estado de las restauraciones, $58 \%$ se encontraron óptimos, $24 \%$ aceptables y $18 \%$ no aceptable. EI TRA es una herramienta útil para el control de la caries dental en poblaciones aisladas o con menor acceso a una atención odontológica.

PALABRAS CLAVE: tratamiento restaurativo atraumático, caries dental, cementos de vidrio ionómero, permanencia.

\section{INTRODUCCIÓN}

La caries dental es un proceso patológico de avance lento, autolimitante y que en ausencia de tratamiento puede avanzar hasta la destrucción del diente (Moncada \& Urzúa, 2008). Esta progresión puede controlarse con técnicas como modificación de hábitos de higiene y dieta, aplicación de flúor tópico y la restauración de las lesiones cariosas (Frencken et al., 2011). Actualmente se ha incorporado el tratamiento restaurador atraumático (TRA o ART por sus siglas en inglés) a la práctica clínica, como un método definitivo o provisional para controlar los altos niveles de caries (Otazú \& Perona, 2005). El TRA es un ejemplo del concepto de la odontología mínimamente invasiva (Tyas et al., 2000), el que ha progresado gracias a los nuevos conocimientos sobre cariología y el desarrollo de nuevos materiales de restauración (van GemertSchriks et al., 2007). Esta filosofía, nace en respuesta a los conceptos tradicionales establecidos por Black sobre el manejo de la caries dental, y pretende mantener la funcionalidad de los dientes por el mayor tiempo posible (van Gemert-Schriks et al.; Frencken et al., 2012). Se basa en un componente preventivo y otro restaurativo; el primero se realiza mediante sellantes de fosas y fisuras, para evitar la formación de nuevas caries; mientras que el segundo componente también incluye al primero, ya que además de obturar la lesión cariosa se realiza el sellado de las fosas y fisuras adyacentes con el mismo biomaterial (van't Hof et al., 2006; Frencken et al., 2011)

La diferencia fundamental entre el TRA y otras intervenciones mínimamente invasivas, es el empleo de instrumental manual (Frencken \& Leal, 2010) combinado con el uso de materiales adhesivos para la res-

\footnotetext{
Alumno Tesista, Facultad de Odontología, Universidad del Desarrollo, Concepción, Chile.

* Cirujano Dentista e Imagenólogo Dentomaxilofacial. Facultad de Odontología, Universidad del Desarrollo, Concepción, Chile.

${ }^{* *}$ Cirujano Dentista, Diplomado en Metodología de la Investigación Clínica. Facultad de Odontología, Universidad del Desarrollo, Concepción, Chile.
} 
tauración de las cavidades manualmente preparadas (Frencken et al., 1996). El material restaurador recomendado y más usado es el cemento de vidrio ionómero, gracias a características como liberación constante de flúor (Lohbauer, 2010), fácil manipulación, biocompatibilidad y buenas propiedades físicomecánicas (Tascón, 2005). Entre las desventajas del ionómero de vidrio se encuentran su relativa aspereza y su resistencia subóptima al agua inmediatamente después de la obturación. Por otro lado, son importantes sus limitaciones estéticas y los riesgos de microfiltración marginal y fractura en cavidades compuestas. Las desventajas anteriores impiden elegir al vidrio ionómero como material restaurativo definitivo en cavidades compuestas (Tascón).

Las restauraciones tipo TRA tienen un tiempo de vida menor a 5 años y se deben emplear principalmente en pacientes con dentición temporal (Bello \& Fernández, 2008). Sin embargo, hay que recalcar que la técnica fue creada para beneficiar primordialmente a personas de nivel socioeconómico bajo, por lo que también puede ser utilizado ante la presencia de caries en dientes permanentes (Studart et al., 2012). Es por esto que se utilizó esta técnica para intervenir en una comunidad de la IX región de Chile, Curarrehue. Esta comuna se encuentra a $112 \mathrm{~km}$ de Temuco, capital regional, y cuenta con una población aproximada de 6.800 habitantes. Según los indicadores es una comuna con alta vulnerabilidad en salud, siendo considerada zona extrema cercana a la cordillera y de difícil acceso en invierno, con una comunidad mayoritariamente mapuche y donde sus principales actividades son la ganadería y agricultura. Presenta un centro de salud ambulatorio y cuatro postas rurales. Todos estos antecedentes respaldaron la decisión de realizar un voluntariado dental llamado Friendship and Oral Health durante el mes de febrero del 2012. Tras la realización de esta actividad se llevó a cabo el estudio y análisis de la estadística para la posterior realización del presente trabajo. Éste tiene como objetivo determinar la permanencia y el estado de las restauraciones ART realizadas en dientes permanentes de niños entre 6 - 16 años.

\section{MATERIAL Y MÉTODO}

Se realizó un estudio descriptivo longitudinal. La población blanco la conformaron 249 niños de 3 a 16 años (Tabla I), atendidos durante febrero del 2012 en el operativo de salud "Friendship and Oral Health" en la comuna de Curarrehue. El tamaño muestral se determinó con un nivel de confianza del 95\%, un error de muestreo máximo del $5 \%$ y considerando una población blanco de 249 niños. Se establecieron criterios de inclusión, los cuales fueron que los niños pertenecieran a la comuna de Curarrehue; presentaran primeros molares permanentes sin sintomatología; tuvieran entre 6 a 16 años; y que hayan recibido atención dental durante el operativo "Friendship and Oral Health", en febrero del 2012. Además se aplicaron criterios de exclusión, donde se exceptuaron a los pacientes que presentaron dientes con sintomatología dolorosa o exposición de la cámara pulpar. Se realizó un muestreo aleatorio entre las fichas que cumplieran estos criterios.

Tabla I. Distribución de la población total de pacientes estudiados.

\begin{tabular}{llcc}
\hline \multirow{3}{*}{ Sexo } & & $\mathbf{n}$ & $\mathbf{\%}$ \\
\cline { 3 - 4 } Edad & Hombre & 123 & 49,4 \\
& Mujer & 126 & 50,6 \\
& $0-4$ años & 26 & 10,44 \\
& $5-8$ años & 89 & 35,74 \\
& $9-12$ años & 89 & 35,74 \\
& $13-16$ años & 45 & 18,07 \\
\hline
\end{tabular}

Se contactó a los padres de los sujetos seleccionados tras el muestreo para explicar la finalidad del estudio y obtener su consentimiento. Se confeccionó y validó por juicio de expertos una ficha clínica, que contenía los criterios establecidos por Frencken y utilizados por Farag et al. (2011), para evaluar permanencia (Tabla II) y estado de los dientes (Tabla III). Luego de 7 meses de realizadas las obturaciones se asistió nuevamente a la comuna intervenida y se realizó un examen clínico a los participantes, donde se evaluaron las restauraciones mediante método visual y táctil por medio de sonda. La evaluación fue realizada por dos operadores previamente calibrados, obteniendo un coeficiente Kappa de 1. Éste se obtiene mediante el test homónimo, que refleja la fuerza de concordancia que existe entre dos observadores al trabajar con variables categóricas (Cerda \& Villarroel, 2008).

Los resultados registrados en las fichas clínicas fueron digitalizados y analizados por medio del programa Microsoft Office Excel, ilustrando la información recopilada en gráficos. La participación de los pacientes contó con el debido asentimiento informado y durante la recolección de datos se resguardaron todos los protocolos sanitarios correspondientes de acuerdo con las normas éticas de la Declaración de Helsinki de 2000. 
Tabla II. Criterios de evaluación para determinar permanencia de las restauraciones.

\begin{tabular}{ll}
\hline Criterio & \multicolumn{1}{c}{ Observación } \\
\hline 0 & Satisfactoriamente presente \\
1 & Presente, pequeña deficiencia en margen menor de $0.5 \mathrm{~mm}$ \\
2 & Presente, deficiencia en margen de $0,5 \mathrm{~mm}$ o más \\
3 & Presente, fractura en restauración \\
4 & Presente, fractura en remanente dentario \\
5 & Presente, sobreextensión del margen aproximado a $0,5 \mathrm{~mm}$ o más \\
6 & Ausente, mayoría de la restauración o su totalidad \\
7 & Ausente, otro tratamiento restaurativo realizado \\
8 & Ausente, diente no está presente \\
9 & Incapaz de determinar \\
C & Caries presente \\
\hline
\end{tabular}

Tabla III. Criterios de evaluación para determinar estado de las restauraciones.

\begin{tabular}{ll}
\hline Criterio & Observación \\
\hline Óptimo & $\begin{array}{l}\text { La restauración se encuentra en óptimas condiciones: buen sellado marginal, sin rasgos de } \\
\text { fractura y/o desgaste de material obturador. }\end{array}$ \\
Aceptable & $\begin{array}{l}\text { La restauración presenta alguna insuficiencia en su estructura sin alterar su funcionamiento: } \\
\text { deficiencia marginal menor a } 0,5 \mathrm{~mm}, \text { cambio de coloración y/o desgaste de material } \\
\text { obturador. }\end{array}$ \\
No Aceptable & $\begin{array}{l}\text { La restauración no se encuentra en condiciones para proteger la estructura dentaria } \\
\text { remanente. }\end{array}$ \\
\hline
\end{tabular}

\section{RESULTADOS}

De un total de 249 niños, se aplicaron los criterios de inclusión y exclusión para conformar una muestra de 65 participantes. Todos aceptaron ser parte del estudio y firmaron el asentimiento informado. Un 55\% correspondía a sexo masculino, y el promedio de edad fue de 10 años. Fueron examinadas 172 dientes, las que fueron tratadas con técnica restaurativa atraumática utilizando el vidrio ionómero Ketac Molar (3M ESPE, St. Paul, MN, US). Estos dientes fueron evaluados 7 meses después de ser tratadas, mediante examen visual y táctil (sonda). El proceso evaluó la permanencia (Fig. 1) y el estado (Fig. 2).

De un total de 172 dientes tratados con restauraciones simples, 142 obturaciones se encontraron presentes, correspondiente a un $82 \%$ de permanencia. De éstas, 99 fueron evaluadas con puntaje ideal y 34 presentaron una deficiencia marginal menor a $0,5 \mathrm{~mm}$, lo que clínicamente se considera correcto. En el resto de las restauraciones presentes se encontraron imperfecciones, como una deficiencia marginal mayor a $0,5 \mathrm{~mm}$, o una fractura de la restauración o del remanente dentario. Del resto de los dientes examinados, 15 restauraciones se encontraban ausentes, ya sea en su mayoría o en su totalidad. En 1 caso, el diente estaba ausente y en 14 se encontró caries, ya sea adyacente a la restauración o en ausencia de ésta.

En relación al estado de las restauraciones, 99 se encontraron óptimas, 42 aceptables y 31 en estado no aceptable. Cabe mencionar que en la práctica clínica, tanto los dientes en estado óptimo como aceptable protegen la estructura dentaria remanente. 


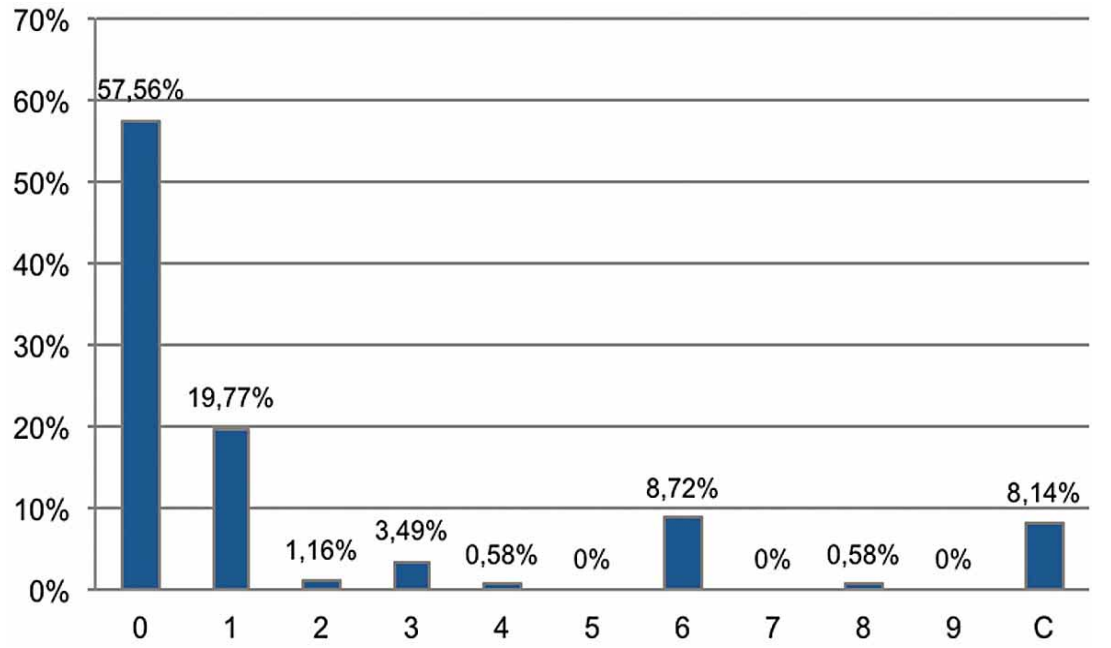

Fig. 1. Porcentaje de dientes de acuerdo a criterios de permanencia.

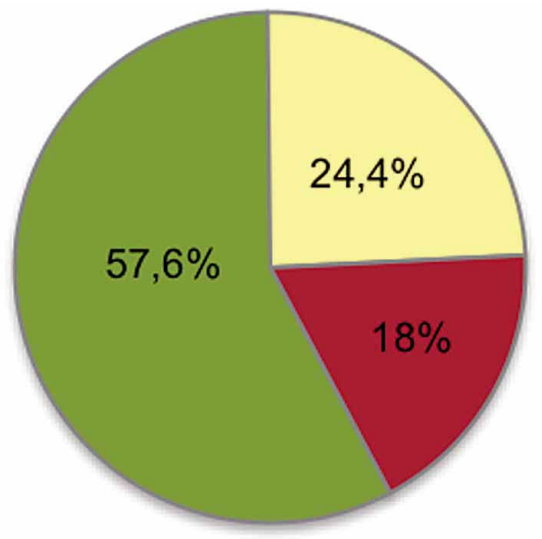

\section{1. Óptimo $\quad$ 2. Aceptable $\quad$ घ3. No aceptable}

Fig. 2. Porcentaje de dientes de acuerdo a criterios de estado de las restauraciones

\section{DISCUSIÓN}

De acuerdo a lo observado, se evidencia que el TRA es una herramienta útil para el control de la caries dental en poblaciones aisladas o con menor acceso a una atención odontológica (Abid et al., 2002). Se observó que más del $80 \%$ de las restauraciones permanecieron en su cavidad después de 7 meses de realizadas. Dentro de estas obturaciones, un $14 \%$ presentó una deficiencia marginal menor a 0,5 $\mathrm{mm}$, lo que es clínicamente aceptable ya que se puede mantener y controlar su estado periódicamente con una buena técnica de higiene. En cuanto al estado de las restauraciones, sobre el $80 \%$ de éstas se encontraban en forma óptima o aceptable, siendo clínicamente funcionales al proteger la estructura dentaria remanente. El resto de las obturaciones presentó deficiencias como desgaste excesivo, cambio de coloración y/o textura, falta de material o presencia de caries.
Los resultados obtenidos en el presente estudio son similares a los obtenidos por de Souza et al. (2003) y Roshan \& Sakeenabi (2011), quienes obtuvieron indicadores de permanencia de $86 \%$ y $85,96 \%$ respectivamente. Estos trabajos evaluaron la permanencia de las restauraciones después de 8 y 6 meses correspondientemente. Por otro lado, los resultados difieren de estudios donde se obtuvieron porcentajes de permanencia superior en un tiempo similar (Dülgergil et al., 2005; Bresciani et al., 2005), encontrándose una supervivencia de $92,4 \%$ y de $97,3 \%$ respectivamente tras seis meses de tratamiento con vidrio ionómero convencional. Estos resultados podrían diferir de los obtenidos en el presente estudio por distintos factores, como el tamaño de la cavidad o mantención de los hábitos de higiene de los pacientes. Lo et al. (2007); y Holmgren et al. (2000) determinaron que las cavidades pequeñas restauradas mediante ART tienen una tasa de permanencia mayor a las realizadas en cavidades más extensas. En el presente estudio no se clasificaron las cavidades de acuerdo a su tamaño, pero puede ser un factor determinante para haber obtenido los resultados ya expuestos. Por otro lado, la mantención de los hábitos de higiene de los pacientes es importante, ya que junto con realizar la obturación de los dientes afectados, se deben enseñar técnicas de higiene (cepillado, uso de seda dental y colutorios) y crear conciencia en los pacientes para que mantengan un buen estado de salud bucal. El uso de una buena técnica de cepillado, junto a una pasta de dientes fluorada, es una medida efectiva para evitar el desarrollo de lesiones cariosas. Además, las últimas revisiones concluyen que la remoción periódica de placa bacteriana desde una lesión dentinaria parece disminuir o evitar la progresión de dicha lesión (Frencken et al., 2012). 
Dentro de las limitaciones que tuvo el estudio, se pueden mencionar las complicaciones para contactar a los participantes, ya que muchos viven en zonas aisladas o no poseen un teléfono de contacto. Por otro lado existieron complicaciones asociadas a la técnica de restauración, como es la ausencia de un sistema de eyección que podría otorgar un campo clínico más idóneo para realizar las obturaciones. Un buen aporte para próximos estudios sería complementar la técnica mediante la implementación de un sistema de eyección y el uso de material rotatorio, tanto para la eliminación de caries como para el posterior ajuste y pulido de la restauración. Además sería de gran utilidad poder contar con radiografías pre y post intervención clínica, para mejorar el diagnóstico y control de las restauraciones oclusales, y para descartar la presencia de caries interproximales. Por último, es posible determinar que el tratamiento restaurador atraumático es una herramienta efectiva y útil para el control de caries, pudiendo ser una alternativa válida para tratar poblaciones aisladas sin un fácil acceso a centros de salud asistencial.

RUDLOFF, T. K.; HARISTOY, O. R. \& VELÁSQUEZ, C. M. Survival of atraumatic restorative treatment (ART) restorations in permanent teeth. Int. J. Odontostomat., 8(1):53-58, 2014.

ABSTRACT: The atraumatic restorative treatment (ART) has been incorporated into clinical practice as a definitive and/or provisional method to control high levels of caries. It is particularly useful when you need to deliver care to isolated communities with limited access to public health care system. The aim of the study was to determine the permanence and status of fillings made with atraumatic restorative technique in permanent teeth of children aged 6-16 years in the commune of Curarrehue, Araucanía Region, Chile. The target population consisted of 249 children between 3 and 16 years living in Curarrehue. Seven months after performing fillings, a clinical examination was performed to participants where restorations were evaluated according to the criteria established by Frencken to evaluate the ART. One hundred seventy-two teeth restored with ART were examined, 142 of these restorations were successful, corresponding to $82 \%$ of permanence. Furthermore, regarding the status of the restorations, $58 \%$ were found to be in optimal condition, $24 \%$ were considered acceptable and $18 \%$ were considered unacceptable. According to observations during the course of this work, it becomes apparent that ART is a useful tool for the control of dental caries in isolated populations or with less access to dental care.

KEY WORDS: atraumatic restorative treatment, dental caries, glass ionomer, survival.

\section{REFERENCIAS BIBLIOGRÁFICAS}

Abid, A.; Chkir, F.; Ben Salem, K.; Argoubi, K. \& SfarGandoura, M. Atraumatic restorative treatment and glass ionomer sealants in Tunisian children: survival after 3 years. East Mediterr. Health J., 8(2-3):315-23, 2002.

Bello, S. \& Fernández, L. Tratamiento restaurador atraumático como una herramienta de la odontología simplificada. Revisión bibliográfica. Acta Odontol. Venez., 46(4):567-72, 2008.

Bresciani, E.; Carvalho, W. L.; Pereira, L.C .; Barata, T. J.; García-Godoy, F. \& Navarro, M. F. Six-month evaluation of ART one-surface restorations in a community with high caries experience in Brazil. J. Appl. Oral Sci., 13(2):1806, 2005.

Cerda, J. \& Villarroel, L. Evaluación de la concordancia interobservador en investigación pediátrica: Coeficiente de Kappa. Rev. Chil. Pediatr., 79(1):54-8, 2008.

de Souza, E .M.; Cefaly, D. F.; Terada, R. S.; Rodrigues, C. C. \& de Lima Navarro, M. F. Clinical evaluation of the ART technique using high density and resin-modified glass lonomer cements. Oral Health Prev. Dent. 1(3):201-7, 2003.

Dülgergil, C. T.; Soyman, M. \& Civelek, A. Atraumatic restorative treatment with resin-modified glass ionomer material: short-term results of a pilot study. Med. Princ. Pract., 14(4):277-80, 2005.

Farag, A.; van der Sanden, W. J.; Abdelwahab, H. \& Frencken, J. E. Survival of ART restorations assessed using selected FDI and modified ART restoration criteria. Clin. Oral Investig., 15(3):409-15, 2011.

Frencken, J. E. \& Leal, S. C. The correct use of the ART approach. J. Appl. Oral Sci., 18(1):1-4, 2010.

Frencken, J. E.; de Amorim, R. G.; Faber, J. \& Leal, S. C. The Caries Assessment Spectrum and Treatment (CAST) index: rational and development. Int. Dent. J., 61(3):11723, 2011.

Frencken, J. E.; Peters, M. C.; Manton, D. J.; Leal, S. C.; Gordan, V. V. \& Eden, E. Minimal intervention dentistry 
for managing dental caries - a review: report of a FDI task group. Int. Dent. J., 62(5):223-43, 2012.

Frencken, J. E.; Pilot, T.; Songpaisany, Y. \& Phantumvanit, $P$. Atraumatic restorative treatment (ART): rationale, technique, and development. J. Public Health Dent., 56(3):135-40, 1996.

Holmgren, C. J.; Lo, E. C.; Hu, D. \& Wan, H. ART restorations and sealants placed in Chinese school children - results after three years. Community Dent. Oral Epidemiol., 28(4):314-20, 2000.

Lo, E. C.; Holmgren, C. J.; Hu, D. \& van Palenstein Helderman, W. Six-year follow up of atraumatic restorative treatment restorations placed in Chinese school children. Community Dent. Oral Epidemiol., 35(5):387-92, 2007.

Lohbauer, U. Dental Glass lonomer Cements as Permanent Filling Materials? -Properties, Limitations and Future Trends. Materials, 3(1):76-96, 2010.

Moncada, G. \& Urzúa, I. Cariología Clínica. Bases Preventivas y Restauradoras. Santiago, Colgate, 2008.

Otazú, C. \& Perona, G. Técnica Restaurativa Atraumática. Conceptos actuales. Rev. Estomatol. Herediana, 15(1):77-81, 2005.

Roshan, N. M. \& Sakeenabi, B. Survival of occlusal ART restorations in primary molars placed in school environment and hospital dental setup-one year followup study. Med. Oral Patol. Oral Cir. Bucal, 16(7):e973-7, 2011.

Studart, L.; da Franca, C. \& Colares, V. Atraumatic Restorative Treatment in permanent molars: a systematic review. Braz. J. Oral Sci., 11(1):36-41, 2012.

Tascón, J. Restauración atraumática para el control de caries dental: historia, características y aporte de la técnica. Rev. Panam. Salud Publica, 17(2):110-5, 2005.

Tyas, M. J.; Anusavice, K. J.; Frencken, J. E. \& Mount, G. J. Minimal intervention dentistry--a review. FDI Commission Project 1-97. Int. Dent. J., 50(1):1-12, 2000.

van Gemert-Schriks, M. C.; van Amerongen, W. E.; ten Cate, J. M. \& Aartman, I. H. Three-year survival of single- and two-surface ART restorations in a high-caries child population. Clin. Oral Investig., 11(4):337-43, 2007.

van't Hof, M. A.; Frencken, J. E.; van Palenstein Helderman, W. H. \& Holmgren, C. J. The atraumatic restorative treatment (ART) approach for managing dental caries: a meta-analysis. Int. Dent. J., 56(6):345-51, 2006.
Dirección para Correspondencia:

Manuel Velásquez C.

Barros Arana 1735

Concepción

CHILE

Fono-Fax 56-41-2268814

Email: manuelvelasquez@udd.cl.

Recibido: 05-08-2013

Aceptado: 11-11-2013 